\title{
PERFIL EPIDEMIOLÓGICO DAS MULHERES COM DIAGNÓSTICO DE CÂNCER DE COLO UTERINO EM REGIÃO DE ALTA INCIDÊNCIA DO NORTE DO PAÍS NO PERÍODO DE 2014 - 2018
}

EPIDEMIOLOGICAL PROFILE OF WOMEN WITH DIAGNOSIS OF CERVICAL CANCER IN A REGION WITH A HIGH INCIDENCE IN THE NORTH OF THE COUNTRY IN THE PERIOD 2014 - 2018

\author{
Gabriela Marques Pimenta ${ }^{1}$ \\ Giulia Valcanaia Dutra \\ ${ }^{2}$ Angélica Lins Linhares Peixoto Pinheiro ${ }^{3}$
}

RESUMO: OBJETIVO: Câncer do colo do útero (CCU) é o resultado da alteração maligna das células que revestem o colo do útero. $\mathrm{Na}$ análise nacional, o câncer do colo do útero é o primeiro mais incidente na Região Norte (26,24/100 mil). O objetivo do presente trabalho é o estudo do perfil epidemiológico de CCU no Tocantins, de acordo com dados demográficos, noperíodo de 2014-2018. METODOLOGIA: Tratase de um estudo epidemiológico descritivo de casos positivos para câncer de colo de útero, de mulheres naturais e procedentes do estado do Tocantins, através da coleta de dados anuais fornecidos pelo Integrador Registro Hospitalarde Câncer do Instituto Nacional de Câncer (INCA), disponibilizado pelo Tabnet, aplicativo desenvolvido pelo Departamento de Informática do Sistema Único de Saúde (DATASUS). RESULTADOS: Encontrou-se um alto índice de mulheres com baixa escolaridade, pardas, com faixa etária predominante entre 30-54 anos e ocupações de baixa complexidade, diagnosticadas com câncer de colo uterino. CONCLUSÃO: O foco principal da prevenção está na investigação epidemiológica, como uma ferramenta imprescindível para reconhecimento e o entendimento da dinâmica para controle do CCU.

Palavras chave: Saúde feminina. Neoplasias cervicais. Colpocitologia.

\footnotetext{
1 Acadêmica de Medicina no Centro Universitário Presidente Antônio Carlos (UNITPAC), Araguaína - TO.

${ }^{2}$ Acadêmica de Medicina no Centro Universitário Presidente Antônio Carlos (UNITPAC), Araguaína - TO.

${ }^{3}$ Médica. Professora no curso de Medicina no Centro Universitário Presidente Antônio Carlos (UNITPAC), Araguaína - TO.
} 
ABSTRACT: OBJECTIVE: Cervical cancer (CC) is the result of the malignant alteration of the cells that line the cervix. In the national analysis, cervical cancer is the first most incident in the North Region (26.24 / 100 thousand). The aim of this study is to study the epidemiological profile of CC in Tocantins, according to demographic data, in the period 2014-2018. METHODOLOGY: This is a descriptive epidemiological study of positive cases for cervical cancer, of women natural and coming from the state of Tocantins, through the collection of annual data provided by the Integrator Hospital Registry of Cancer of the National Cancer Institute (INCA), made available by Tabnet, an application developed by the Department of Informatics of the Unified Health System (DATASUS). CONCLUSION: The main focus of prevention is on epidemiological investigation, as an essential tool for recognizing andunderstanding the dynamics for controlling CC.

Keywords: Women's health. Cervical neoplasms. Colpocytology. 


\section{INTRODUÇÃO}

Câncer do colo do útero (CCU) é o resultado da alteração maligna das células que revestem o colo do útero: as células espinocelulares (na exocérvice) e as células glandulares (na endocérvice). $O$ processo ocorre quando essas células sofrem alterações anormais de forma gradativa, aparecendo primeiramente as lesões précancerígenas. Há duas categorias principais de carcinoma invasores do colo uterino: o carcinoma epidermoide, mais comum, acomete o epitélio escamoso (cerca de $80 \%$ dos casos), e o adenocarcinoma, mais raro e que acomete o epitélio glandular. (BRASIL, 2013).

O câncer in situ, ou não invasivo, tem esse nome pois não ultrapassou a membrana basal, que é uma barreira que divide a camada superficial da pele e das mucosas, das camadas mais profundas, onde se encontram os vasos sanguíneos e linfáticos. Nessa camada não há vasos sanguíneos portanto a chance da doença se espalhar é extremamente baixa. Quando a doença ultrapassa a membrana basal é chamada de câncer invasivo, ou infiltrativa. Nesse momento a doença pode causar metástase a distância. (ADES,2016).

É uma doença de desenvolvimento lento, que pode cursar sem sintomas em fase inicial e evoluir para quadros de sangramento vaginal intermitente ou após a relação sexual, secreção vaginal anormal e dor abdominal associada com queixas urinárias ou intestinais nos casos maisavançados. (INCA, 2021).

As taxas de incidência estimadas e de mortalidade no Brasil apresentam valores intermediários em relação aos países em desenvolvimento, porém são elevadas quando comparadas às de países desenvolvidos com programas de detecção precoce bem estruturados.(INCA,2021).

$\mathrm{Na}$ análise regional do país, o câncer do colo do útero é o primeiro mais incidente na Região Norte (26,24/100 mil) e o segundo nas Regiões Nordeste $(16,10 / 100$ mil) e Centro- Oeste (12,35/100 mil). Já na Região Sul (12,60/100 mil), 
ocupa a quarta posição e, na Região Sudeste (8,61/100 mil), a quinta posição (INCA, 2021).

Quanto à mortalidade, é também na Região Norte que se evidenciam as maiores taxas do país, sendo a única com nítida tendência temporal de crescimento. Em 2019, a taxa padronizada pela população mundial foi de 12,58 mortes por 100.000 mulheres, representando a primeira causa de óbito por câncer feminino nesta região. Nas Regiões Nordeste com taxa demortalidade de 6,66/100 mil, foi a segunda causa e Centro-Oeste, a terceira causa, com taxa de 6,32/100 mil. As Regiões Sul e Sudeste tiveram as menores taxas (4,99/100 mil e 3,71/100 mil) representando a quinta e sexta posições, respectivamente, entre os óbitos por câncer em mulheres. (INCA, 2021).

O câncer cérvico-uterino vem sendo correlacionado com diversos elementos causais ao longo dos tempos. Atualmente são conhecidos os seguintes fatores de risco para leões cervicais: DST; condições infecciosas e reativas; hábitos sexuais, como início precoce e multiplicidade de parceiros; tabagismo ativo e passivo; uso prolongado de anticoncepcionais orais. Carências nutricionais, receio da cliente em realizar o exame devido ao medo, vergonha, ansiedade, ignorância e dificuldade de acesso aos serviços de saúde para realização de exame preventivo podem ser considerados como fatores que dificultam o diagnóstico precoce. Sabe-se ainda que por meio dos exames preventivos periódicos pode-se controlar a doença, rastreando a população sintomática e assintomática, levando, na maioria dos casos, à cura. (BEZERRA et al., 2005).

Muitos fatores podem contribuir para explicar o sucesso apenas parcial dos programas de rastreamento na região Norte do Brasil, como: peculiaridades culturais dos povos nativos, isolamento geográfico, limitações inerentes à própria técnica do teste de Papanicolaou, falhas no acompanhamento das lesões pré-malignas e adoção de condutas inadequadas. A cobertura populacional da estratégia preventiva é um fator crucial nesse processo. No Brasil, aspectos relacionados à oferta e acesso aos sistemas de saúde têm sido amplamente estudados eapontados como etapa limitante para o controle do CCU em diversas regiões. (NAVARRO et al., 2015). 
Partindo desse pressuposto, o objetivo do presente trabalho é o estudo do perfil epidemiológico das mulheres com câncer de colo uterino, nascidas e tratadas no Tocantins, estado da Região Norte com grande incidência da doença. Diante do número crescente de casos, principalmente no interior do estado, se mostra eficaz a obtenção de dados estatísticos, fonte de informação para pesquisas e planejamento de ações estratégicas em saúde para o controle e prevenção à essa morbidade.

\section{MÉTODOS}

Trata-se de um estudo transversal, realizado nos hospitais de referência do estado do Tocantins, com a utilização de dados de fonte secundária. Os referidos hospitais são instituições de nível terciário, que se caracterizam como de grande porte, de alta complexidade, sendo referências para o estado do Tocantins (TO), composto por 1.572 .866 habitantes. Além disso, foi realizado uma revisão bibliográfica afim de analisar, avaliar e discutir os resultadosencontrados.

Para a coleta dos dados, foi utilizado o banco de dados do Registro Hospitalar de Câncer (RHC), que fornece informações, para o Registro de Câncer de Base Populacional (RCBP), queconstitui um importante instrumento de apoio à formulação da Política Nacional de Atenção Oncológica, ao planejamento de saúde, à avaliação da qualidade da atividade assistencial e como recurso para a elaboração de pesquisas clinicas e trabalhos científicos. Todos os dados que constam no RHC são provenientes dos prontuários de mulheres que receberam o diagnóstico de câncer de colo de útero.

A população de estudo foi composta por todos os casos de CCU do estado do Tocantins no período de 2014 à 2018, de pacientes naturais, residentes e procedentes do estado. Os casosde câncer de colo de útero foram identificados, no banco de dados, por meio do CID-10 (Classificação Internacional de Doenças), sendo o C53 referente à essa neoplasia. Foram identificadas 358 mulheres diagnosticadas com câncer de colo de útero, as variáveis investigadas foram: idade, raça/cor, escolaridade e ocupação. A partir dessas informações selecionadas, foi 
feita uma análise descritiva simples e os dados mais significativos apresentados em gráficos e tabelas.

Por se tratar de um estudo que utiliza dados dos Registros Hospitalares de Câncer (RHC), uma plataforma online do Ministério da Saúde, na qual os mesmos estão disponíveis para livre acesso, o estudo não necessitou de aprovação do comitê de ética em pesquisas em seres humanos.

\section{RESULTADOS E DISCUSSÃO}

Apresentamos os dados obtidos por meio da pesquisa realizada com base no sistema Integrador RHC desenvolvido pelo INCA, 358 mulheres com neoplasia colocervical entre os anos de 2014 a 2018.

Dos 358 casos analisados, os resultados apontam que a predominante foi de 30 a 54 anos, correspondendo a 231 (64,5\%) mulheres. Foi analisada a faixa etária, fora da recomendação do Ministério da Saúde, a qual demonstrou que 7 (1,9\%) da população do estudo com diagnóstico do câncer de colo de útero estava na faixa de 15 a 24 anos e $69(19,2 \%)$ foram diagnosticadas acima dos 65 anos, somando um total de $74(24 \%)$ de mulheres diagnosticadas, fora do período recomendado pelo programa, conforme Tabela 1. 
Tabela 1 - Número de casos segundo faixa etária

\begin{tabular}{|cc|}
\hline Faixa etária & C53 COLO DO UTERO \\
\hline TOTAL & 358 \\
\hline $15-19$ & 2 \\
\hline $20-24$ & 5 \\
$25-29$ & 23 \\
\hline $30-34$ & 46 \\
\hline $35-39$ & 66 \\
\hline $40-44$ & 43 \\
\hline $45-49$ & 33 \\
\hline $50-54$ & 43 \\
\hline $55-59$ & 12 \\
\hline $60-64$ & 16 \\
\hline $65-69$ & 31 \\
\hline $70-74$ & 20 \\
\hline $75-79$ & 13 \\
\hline $80-84$ & 3 \\
\hline $85+$ & 2 \\
\hline
\end{tabular}

FONTE: DATASUS.

Essa informação indica uma necessidade de revisão do programa de rastreio do Ministério da Saúde do câncer de colo uterino, visto que a faixa etária recomendada para realização dos exames citopatológicos preventivos é de 25 à 64 anos. Contudo, pode-se observar casos positivos em mulheres com idade inferior e superior à esta, indicando uma necessidade de rastreio ampliado, em toda a vida sexual da mulher.

Segundo uma pesquisa realizada por Navarro et al. (2015) o intervalo de idade que exprime uma maior adesão ao citopatológico é entre 20 a 34 anos e a menor, de 50 a 54 anos. As principais razões referentes a efetuação do preventivo foram: gravidez, rotina periódica, e queixas ginecológicas. Tais achados justificam o resultado do presente estudo e nos desperta para a necessidade de instituir estratégias de prevenção do câncer do colo do útero voltadas para o grupo de mulheres, que encontra-se mais vulnerável a essa enfermidade pelo fato de descuidarem da sua prevenção. 
Ao examinar outro estudo realizado com 906 mulheres, no período de 20002013, no estado de São Paulo, pode-se notar uma semelhança da faixa etária predominante, o qual se deu de 31 à 60 anos, correspondendo a $534(58,9 \%)$ mulheres (FAVARO et al., 2019). Vale ressaltarque o câncer de colo de útero é raro em mulheres até 30 anos, e sua incidência eleva-se, progressivamente, até ter seu pico na faixa de 45 a 50 anos, o que aponta o aumento da idade, como um importante fator de risco para essa neoplasia. (INCA, 2021).

Os casos de câncer em mulheres, como consta na Figura 1, analisados conforme a raça, evidenciou-se que a predominância foi de 257 (72\%) casos de pardas, $21(21 \%)$ eram brancas, $13(4 \%)$ eram pretas, $9(2 \%)$ indígenas e $13(21 \%)$ não tinha sido notificadas.

Figura 1- Número de casos segundo raça/cor.

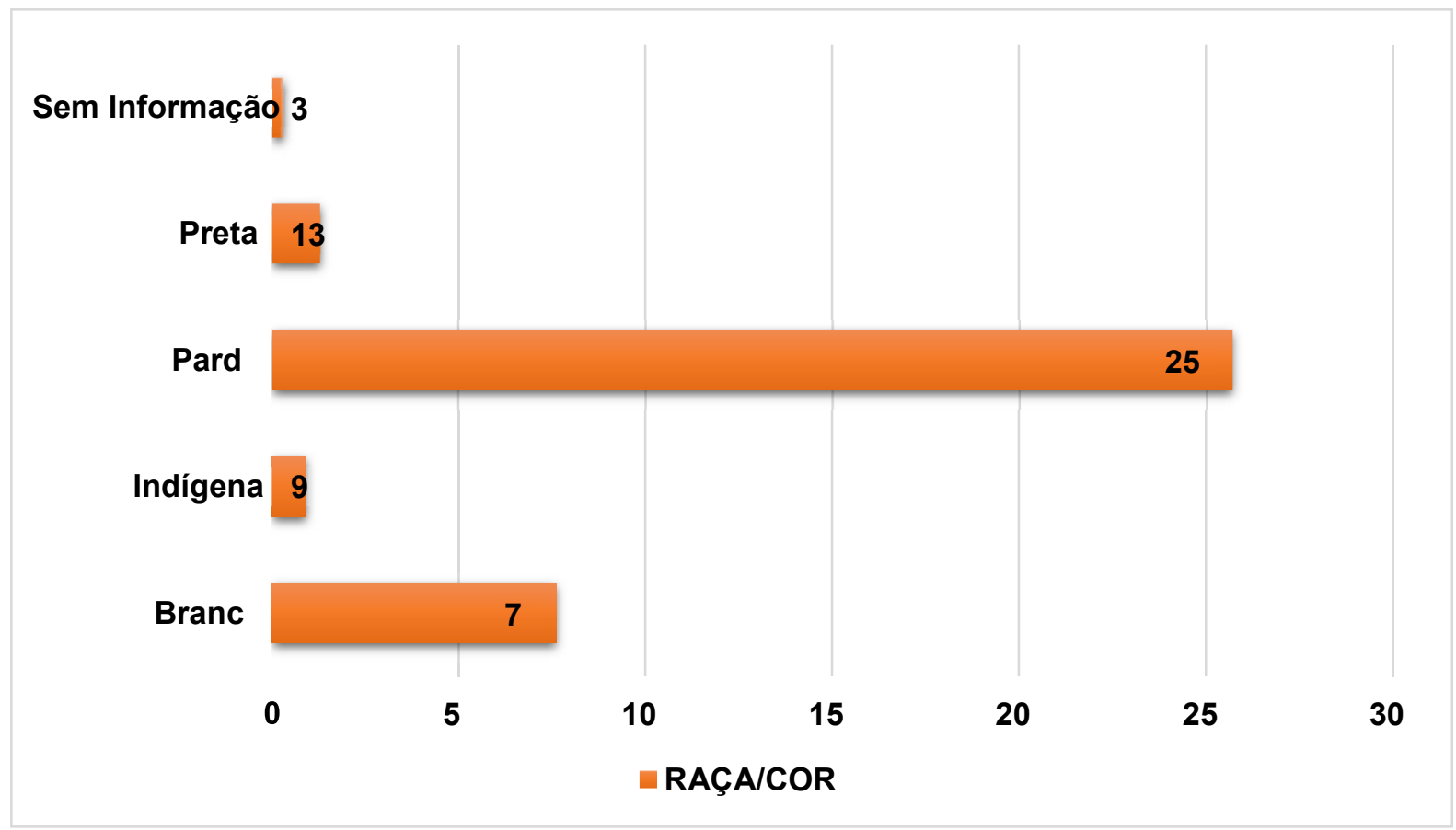

FONTE: INCA, 2021.

Dessa forma, mulheres pardas apresentaram o maior número de câncer uterino no Estado de Tocantins (72\%). Tal fato, comparado com um estudo a nível nacional, realizado entre os anos 2000 à 2009, com total de 77.317 mulheres, se 
assemelha ao resultado deste, poishouve um predomínio de mulheres de cor parda, 26.632 (48\%) (THULER; BERGMANN; CASADO, 2012).

Estudos mostram que um dos fatores primordiais para a diminuição da adesão e prevenção do CCU é o nível de escolaridade, exibindo uma forte associação entre lesão intraepitelial cervical e escolaridade inferior ao ensino fundamental (BORGES et al., 2012; SOARES et al., 2010). Outro fator preponderante que se destaca para a não aderência do examepreventivo é a aversão de ir ao médico realizar o exame, a insegurança de se expor, o fato de acreditar ser desnecessário, a falta de tempo ou ainda o desinteresse. (NAVARRO et al., 2015).

Foi possível confirmar essa narrativa com os dados do estado do Tocantins, o qual 153 pacientes diagnosticadas não possuem ensino fundamental completo, que equivale a $42,7 \%$ dototal de mulheres com câncer de colo uterino, seguido de nível médio completo $(25,7 \%)$, ensino fundamental completo $(13,4 \%)$ e nenhuma escolaridade (12\%). Mulheres com nível superior incompleto $(1,6 \%)$ e completo $(1,9 \%)$ apresentam taxas consideravelmente menores, o que ressalta a associação entre escolaridade e o câncer de colo de útero. Essas informações constamna Figura 2.

Figura 2 - Número de casos segundo escolaridade.

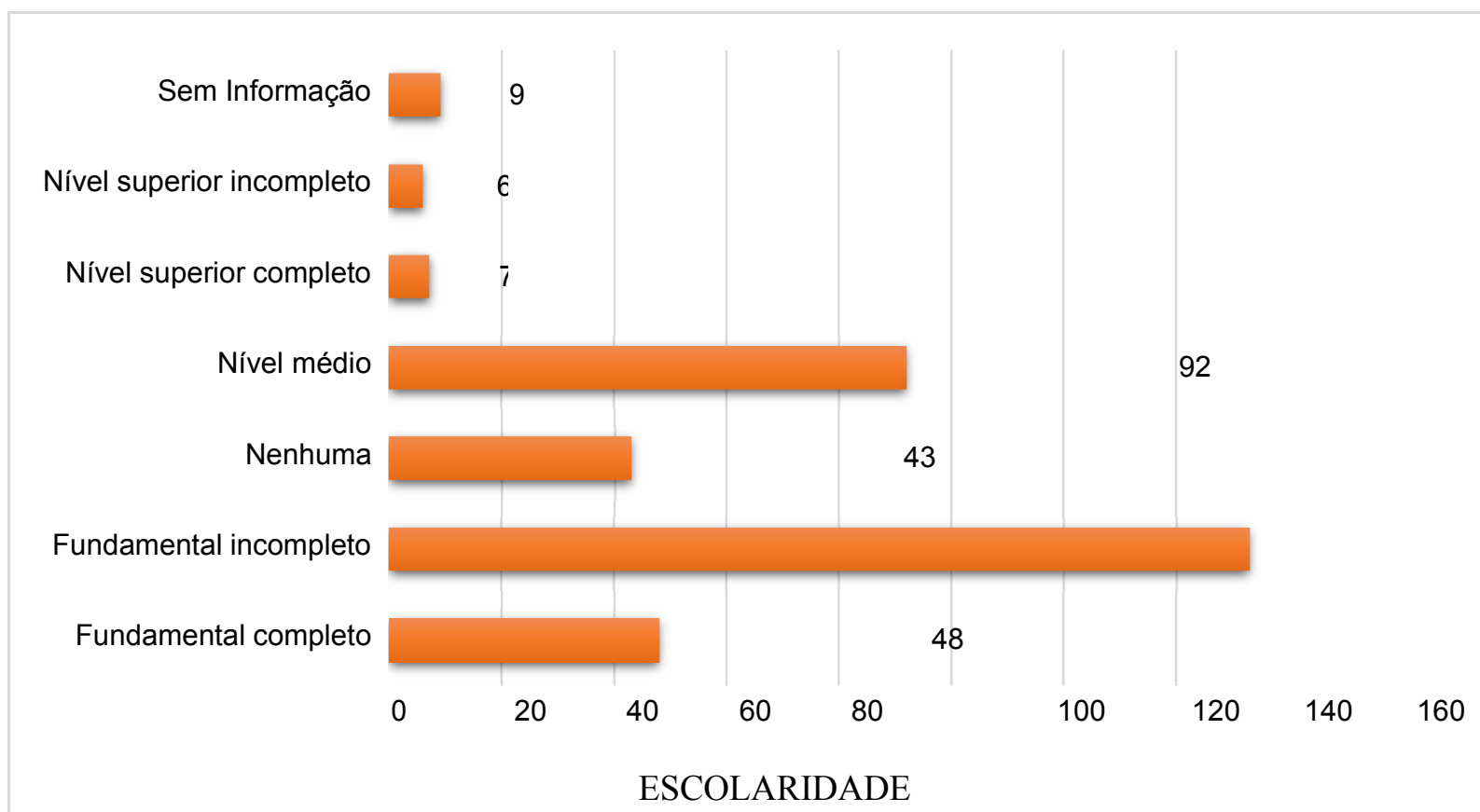

FONTE: INCA, 2021. 
A respeito dos dados obtidos no Registo Hospitalar de Câncer, pelo site do INCA, sobre a ocupação da população estudada, notou-se que a generalidade dos cargos são de trabalhos que não há necessidade de um nível escolar alto concluído. Entre eles, destacam-se: prestadores de serviços gerais (zelador, recepcionista, doméstica, etc.) com 4,5\%, trabalhadores rurais/agropecuários totalizaram em $26,2 \%$ e, em massa, os trabalhadores não classificados segundo ocupação pelo Conselho Brasileiro de Ocupação (CBO), indicando 44,7\%. Esse fato poderia confirmar a correlação da escolaridade e a prevalência do câncer de colo uterino, que pode-se concluir como um dos principais fatores de risco para essa doença.

Outra condição relevante consiste no fato de que, a mulher na sociedade contemporânea,exprime o papel de ser o pilar econômico da família, levando muitas vezes, ao desmazelo coma própria saúde, assim, essas mulheres deixam de buscar os serviços de saúde para realizar o exame. Adicionalmente outros aspectos que foram considerados impasses para a efetividade do exame foram: impedimento de acesso as unidades de saúde, como distância até a unidade, horários não flexíveis e falta de estrutura dos serviços, assim como, o estorvo de agendar um horário com o profissional de saúde (NASCIMENTO et al., 2015).

\section{CONCLUSÃO}

As informações apresentadas demonstram como o câncer colo-cervical ainda é um grave problema de saúde no país, em especial no Tocantins, assim como em toda Região Norte. Os dados compilados sinalizam que o perfil das brasileiras atingidas correspondem em sua maioriamulheres na faixa etária entre 55-59, de cor parda e com baixa escolaridade.

Há uma forte ligação com a escolaridade baixa e a prevalência do câncer de colo uterino, mostrando a relevância em melhorar as estratégias de planejamento e/ou incremento de ações específicas para promoção de saúde e prevenção, com campanhas de alertas e orientações, alémde atividades frequentes de educação em 
saúde considerando grupos/situações de risco, para diminuir incidência no país e, assim, contribuir para melhorar a qualidade de vida.

É necessário também que os profissionais de saúde considerem as particularidades e peculiaridades referentes a aspectos intrínsecos da Região Norte, como a miscigenação do nosso povo, as diferenças locorregionais que impactam a qualidade e o estilo de vida das mulheres e as diferentes condições de acesso aos serviços de saúde. Desta forma, iremos possuirum método de rastreamento que seja realmente eficaz na promoção de saúde e prevenção do câncer colo-cervical.

\section{REFERÊNCIAS BIBLIOGRÁFICAS}

BEZERRA, S. et al. Perfil de mulheres portadoras de lesões cervicais por HPV quanto aos fatores de risco para câncer de colo uterino. DST- J bras Doenças Sex. Transm. 17(2): 143148, 2005.

FRANCESCHINI, J.; SCARLATO, A; CISI, M.C. Fisioterapia nas Principais Disfunções Sexuais Pós-Tratamento do Câncer do Colo do Útero: Revisão Bibliográfica. Rev. Brasil. de Cancerologia, v.56, n.4, p. 501-506, 2010.

FAVARO, Caroline Ribeiro Pereira et al. PERFIL EPIDEMIOLÓGICO DE MULHERESCOM CÂNCER DE COLO DE ÚTERO TRATADAS EM HOSPITAL TERCIÁRIO. Revista de Enfermagem do Centro-Oeste Mineiro, v. 9, 2019.

Instituto Nacional de Câncer (INCA). Controle do câncer de colo de útero: conceito e magnitude. Disponível em: <https://www.inca.gov.br/controle-do-cancer-do-colo-doutero/conceito-e-magnitude>. Acesso em: 20 fev. 2021.

Instituto Nacional de Câncer (INCA). Registro Hospitalar de Câncer. Disponível em: <https://irhc.inca.gov.br/RHCNet/>. Acesso em: 20 fev. 2021.

NASCIMENTO, Gabriel Winston de Carvalho et al. Cobertura do exame citopatológico do colo do útero no Estado de Minas Gerais, Brasil, no período entre 2000-2010: um estudo a partirdos dados do Sistema de Informação do Câncer do Colo do Útero (SISCOLO). Cad. saúde colet., Rio de Janeiro, v. 23, n. 3, p. 253-260, Sept. 2015.

NAVARRO, C. et al. Cobertura do rastreamento do câncer de colo de útero em região de alta incidência. Rev. Saúde Pública. Vol. 49, n.17, 2015.

RANGEL, Gabriela; LIMA, Luciana Dias de; VARGAS, Eliane Portes. Condicionantes do diagnóstico tardio do câncer cervical na ótica das mulheres atendidas no Inca. Saúde debate, Rio de Janeiro, v. 39, n. 107, p. 1065-1078, Dec. 2015.

SOARES, M. B. O.; SILVA, S. R. da. Análise de um programa municipal de prevenção do câncer cérvico-uterino. Brasília. Rev. Brasil. de Enfer. 63 (2): 177-82, mar-abr, 2010.

THULER, Luiz Claudio Santos; BERGMANN, Anke; CASADO, Letícia. Perfil das Pacientescom Câncer do Colo do Útero no Brasil, 2000-2009: Estudo de Base Secundária. Revista Brasileira de Cancerologia, p. 351-357, 2012. 